\title{
Correction: Potent and specific MTH1 inhibitors targeting gastric cancer
}

Wenjuan Zhou, Liying Ma, Jing Yang, Hui Qiao, Lingyu Li, Qian Guo, Jinlian Ma, Lijuan Zhao, Junwei Wang, Guozhong Jiang, Xiangbin Wan, Mariusz Adam Goscinski, Lina Ding, Yichao Zheng, Wencai Li, Hongmin Liu, Zhenhe Suo and Wen Zhao

\section{Correction to: Cell Death \& Disease} https://doi.org/10.1038/s41419-019-1665-3, published online 4 June 2019

Since online publication of this article, the authors noticed that there were errors in Figs. 4 and 5. In Fig. 4, the colouring was omitted from the key for Fig. 4d, g, h, l and $\mathrm{m}$, and an incorrect image was used for OGG1 during the compilation of Fig. 4d. In Fig. 5, incorrect images were used for GAPDH and Procaspase 3 in Fig. 5i, 5j, respectively. The corrected Figures are provided below. The authors confirm that these errors do not affect the conclusions of the article. The authors apologise for any inconvenience this may have caused.

Published online: 16 October 2020 


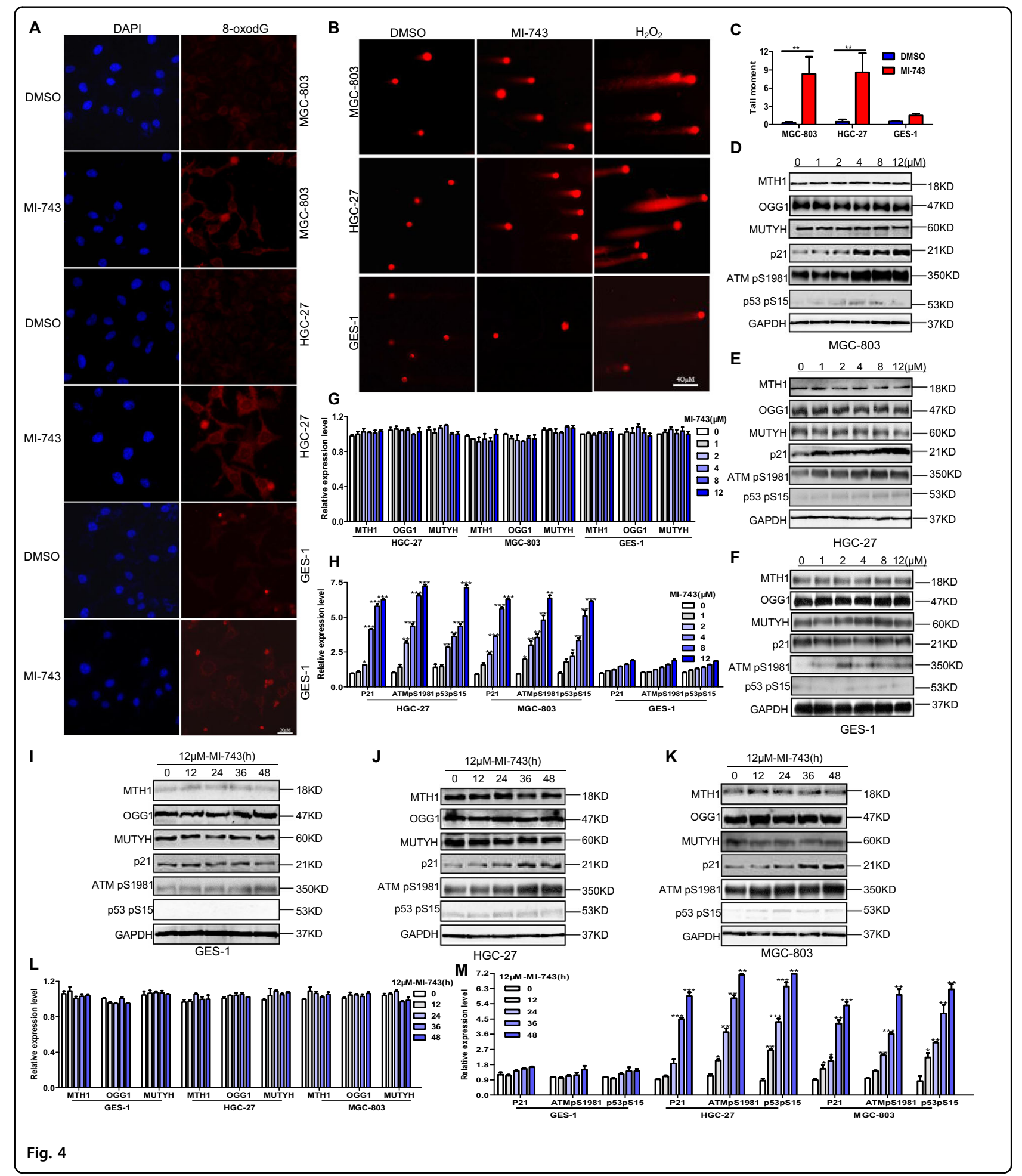




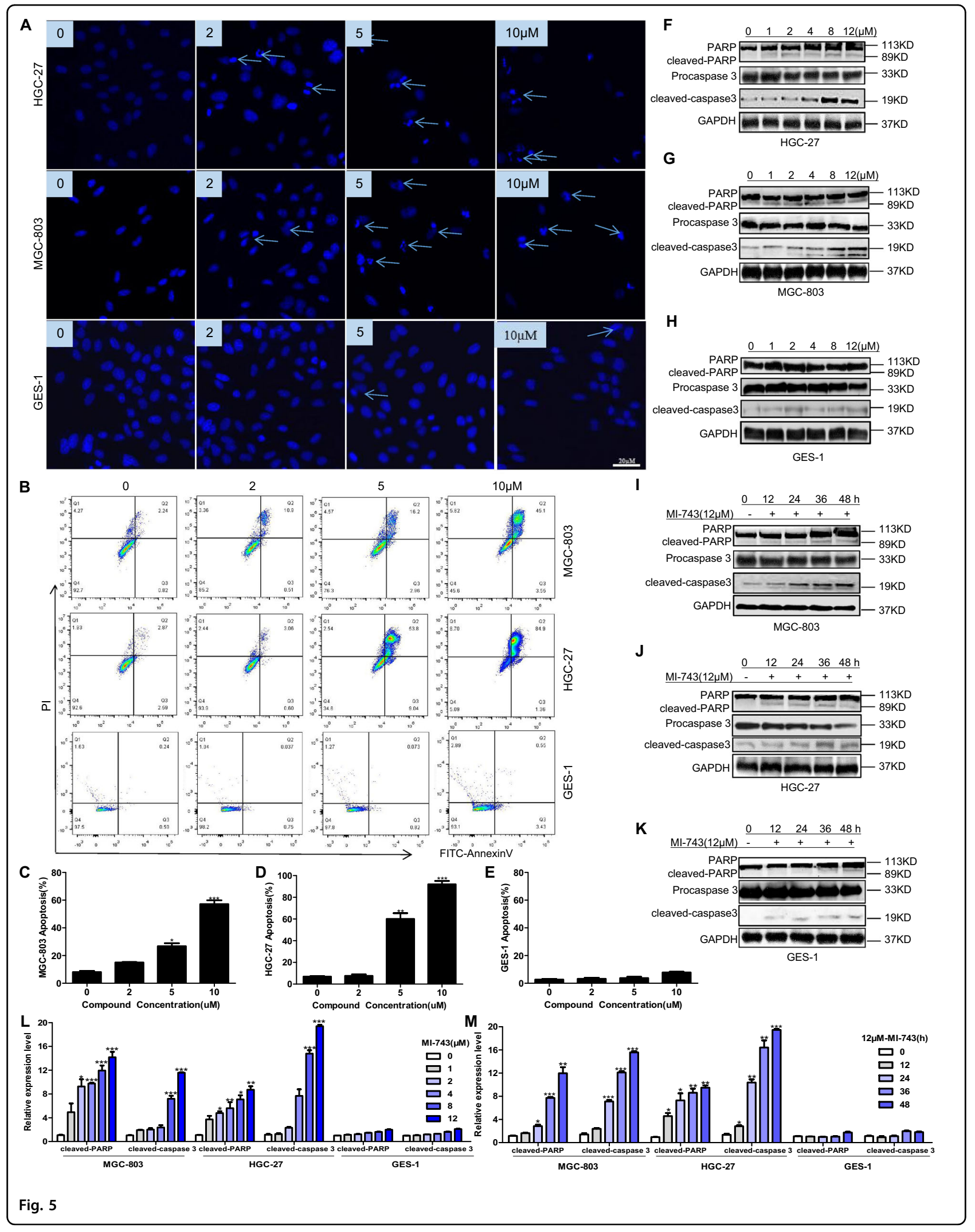

